\title{
Theoretical evidence for strong correlations and incoherent metallic state in FeSe
}

\author{
Markus Aichhorn, ${ }^{1}$ Silke Biermann, ${ }^{1,2}$ Takashi Miyake, ${ }^{2,3,4}$ Antoine Georges, ${ }^{1,2,5}$ and Masatoshi Imada ${ }^{2,4,6}$ \\ ${ }^{1}$ Centre de Physique Théorique, École Polytechnique, CNRS, 91128 Palaiseau Cedex, France \\ ${ }^{2}$ Japan Science and Technology Agency, CREST, Kawaguchi 332-0012, Japan \\ ${ }^{3}$ Research Institute for Computational Sciences, AIST, Tsukuba 305-8568, Japan \\ ${ }^{4} J a p a n$ Science and Technology Agency, TRIP, Kawaguchi 332-0012, Japan \\ ${ }^{5}$ Collège de France, 11 place Marcelin Berthelot, 75005 Paris \\ ${ }^{6}$ Department of Applied Physics, University of Tokyo, Bunkyo-ku, Tokyo 113-8656, Japan
}

\begin{abstract}
The role of electronic Coulomb correlations in iron-based superconductors is an important open question. We provide theoretical evidence for strong correlation effects in FeSe, based on dynamical mean field calculations. Our $a b$ initio spectral properties first demonstrate the existence of a lower Hubbard band. Moreover, together with significant orbital-dependent mass enhancements, we find that the normal state is a bad metal over an extended temperature range, implying a non-Fermi liquid due to formation of local moments. Predictions for angle-resolved photoemission spectroscopy are made.
\end{abstract}

PACS numbers: 71.27.+a, 74.70.Xa, 74.25.Jb

\section{INTRODUCTION}

The discovery of high-temperature superconductivity in iron-based compounds triggered a tremendous amount of experimental and theoretical research. In general terms one can divide these materials into two classes. The first is based on iron-pnictogen $\mathrm{FePn}^{-1}$ planes, which have to be intercalated with some spacer atoms. The second class is built up by charge-neutral iron-chalcogenide FeCn layers, where Cn can be S, Se, or Te. Superconductivity in this ' 11 ' family was first reported by $\mathrm{Hsu}$ et al.,$\frac{1}{\underline{1}}$ with a transition temperature of $T_{c} \approx 8 \mathrm{~K}$ for $\alpha$ $\mathrm{FeSe}_{0.85}$. The properties of this material can be further modified by pressure, ${ }^{2-4}$ excess Fe or Se deficiencies. Another interesting route is alloying FeSe and FeTe yielding the family of $\mathrm{FeSe}_{x} \mathrm{Te}_{1-x}$ superconductors ${ }^{5}$.

In contrast to the high-temperature superconducting cuprates, whose parent compounds are Mott insulators, the question about the strength of correlations in the iron-based superconductors is not settled. FeSe $1-x$ has been studied with angular-integrated photoemission (PES) in Refs. 6, 7], while angular-resolved photoemission studies (ARPES) have been performed on $\mathrm{Fe}_{1+x} \mathrm{Te}^{8}$ and $\mathrm{FeSe}_{x} \mathrm{Te}_{1-x}$ (with $x=0.3$ in [9] and $x=0.42$ in [10]). For all these compounds, these experiments reveal a significant iron bandwidth narrowing, by a factor in excess of $\sim 2$. Interestingly, recent ARPES experiments on $\mathrm{FeSe}_{x} \mathrm{Te}_{1-x}$ with $x=0.42^{10}$ report large mass enhancements deduced from the low-energy Fermi velocities, ranging from 6 to 23, while a smaller enhancement (in the range $2-3$ ) was reported for $\mathrm{Fe}_{1+x} \mathrm{Te}^{8}$. Specific heat measurements ${ }^{11}$ for $\mathrm{FeTe}_{0.67} \mathrm{Se}_{0.33}$ gave $\gamma=39 \mathrm{~mJ} / \mathrm{molK}^{2}$, whereas for $\mathrm{FeSe}_{0.88}$ a smaller value $\gamma=9.17 \mathrm{~mJ} / \mathrm{molK}^{2}$ was reported $\stackrel{\underline{1}}{ }$

Band structure calculations based on densityfunctional theory (DFT) of FeSe and FeTe have shown 12 that the one-electron band structure of these materials is similar to the other iron-based superconductors, as regards the Fe- $d$ states around Fermi level. However, a re- cent first-principles calculation of the screened Coulomb interactions gave significantly higher values for the 11 compounds 13 Dynamical mean-field (DMFT) calculations at $T=0$ - albeit within the iterative perturbation theory - could reproduce some aspects of the experimental results, by taking the interaction as a parameter 14 Given the experimental controversies, further insight from ab initio theoretical predictions, in comparison with experiments, is needed in order to characterize the role of correlations for the 11 family relative to other iron superconductors (e.g. the 1111 family as LaFeAsO).

In this article, we investigate the correlation effects on the electronic structure of $\alpha$-FeSe in the framework of DMFT, using numerically exact Monte Carlo simulations and taking into account the full rotationally invariant Coulomb interactions, evaluated from first principles. We find that this material displays clear evidence of strong correlations. First, our results demonstrate that the satellite feature observed in PES at a binding energy of about $-2 \mathrm{eV}^{\underline{6}, 7}$ should be interpreted as a lower Hubbard band of iron origin. Second, we find that three of the iron orbitals are characterized by a rather low quasiparticle weight, and short quasiparticle lifetimes. These features indicate deviation from conventional Fermi liquid behavior possibly associated with the formation of local moments controlled by the Hund coupling. The possible occurence of local moments in multiband systems was discussed in a model calculation 15 and for 1111 pnictides $\frac{16,17}{}$ for parametrized interaction strengths. The situation in the 1111 is subtle. However, taking the interaction strenght calculated from first priciples, LaFeAsO shows moderate correlations 18 Here, on the contrary, our present calculations with ab initio interactions strongly suggest that correlation effects are most clearly revealed in the 11 family. We make theoretical predictions for the ARPES spectrum of FeSe. Significant deviations from the DFT band-structure are found, which do not simply amount to an overall bandwidth narrowing. A downward shift of the hole-like bands near the 
$\Gamma$-point and an upward shift of the electron-like bands near the M-point are found. Although ARPES has only been reported at this stage for $\mathrm{FeSe}_{x} \mathrm{Te}_{1-x}$, this is consistent with the trends reported for these alloys. 9.10

\section{METHODS}

Our calculations use the recent implementation of the combined DFT-DMFT method in a full-potential augmented plane wave electronic-structure framework ${ }^{18}$ based on the Wien $2 \mathrm{k}$ package $\stackrel{19}{\underline{19}}$ Localized Wannier-like orbitals are constructed from an energy window comprising the Fe- $3 d$ bands and the Se- $4 p$ bands, calculated in the local-density approximation (LDA). We use the tetragonal crystal structure, space group $P 4 / \mathrm{nmm}$, as reported by Margadonna et al.,$\stackrel{20}{\underline{n}}$ using the experimental value for the Se position in the unit cell. A many-body self-energy, computed from DMFT using a strong-coupling continuous-time quantum Monte Carlo algorithm, $\stackrel{21}{2}$ is applied to the subspace spanned by the Fe-3d orbitals. The matrix of Coulomb interaction parameters is calculated from first principles using the con-

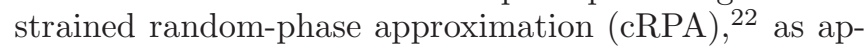
plied to the iron-based superconductors in Refs. 13,23]. All screening transitions are included, except the ones within the Fe-3d manifold. In the notation of Refs. 13, 23 this corresponds to the so-called $d-d p$ construction. In order to avoid an orbital dependent double-counting correction, we do not use the calculated interaction matrices directly. Instead, we follow the procedure described in Ref. [18] and use the orbitally-averaged Coulomb interactions. For FeSe, this yields a local Coulomb integral $U=F_{0}=4.06 \mathrm{eV}$ and a Hund's coupling $J=0.91 \mathrm{eV}$. This is to be compared with the significantly smaller values $U \simeq 2.7 \mathrm{eV}, J \simeq 0.8 \mathrm{eV}$ for $\mathrm{LaFeAsO} \stackrel{13,18}{1}$ Please not that the definition of $J$ is used here in terms Slater integrals, and hence differs from the definition of $J$ in Refs. [13,23]. We will show that the inclusion of the full rotationally-invariant Hund's coupling (including spinflip and pair-hopping) is crucial for FeSe. If not otherwise specified, all calculations were performed at a temperature $T=290 \mathrm{~K}$ using the fully-localized-limit double counting 24 Spectra were obtained from the imaginaryfrequency Monte Carlo data using the stochastic Maximum Entropy method. $\stackrel{25}{ }$

\section{RESULTS}

In Fig. 1 we compare the total momentum-integrated spectral function obtained within LDA and within DMFT. A substantial bandwidth reduction is obtained for the Fe- $d$ states near the Fermi energy. Furthermore, the Se- $p$ ligand states are shifted to larger binding energies as compared to LDA. In addition, there is a structure appearing at a binding energy between -1 and $-2 \mathrm{eV}$. In order to identify the physical nature of this peak, we

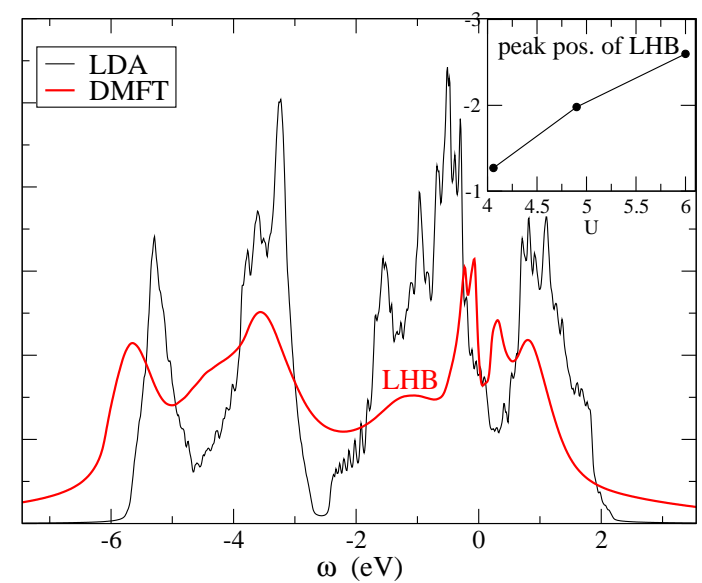

FIG. 1: (Color online) Comparison of the total LDA DOS (black) to the spectral function obtained with DMFT (red). 'LHB' denotes the lower Hubbard band. The inset shows the evolution of the LHB as a function of $U$, with $J$ scaled accordingly $(J=0.9,1.1,1.3$, respectively.)

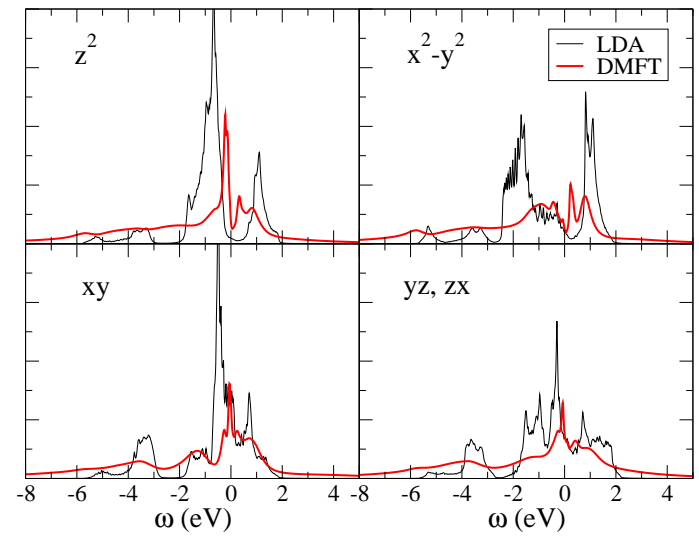

FIG. 2: (Color online) Orbital-resolved comparison of the density of states between the LDA (thin black) and DMFT (thick, red) results. The orbitals in the bottom row show strongest correlations. Note the LHB in the $d_{x y}$ orbital.

performed calculations for larger interactions. For computational efficiency, the calculations for varied $U$ and $J$ were done here with density-density interactions only. Non density-density terms affect mostly the quasiparticle states and little the LHB. It is obvious from the inset of Fig. 1 that the peak shifts towards larger binding energies with increasing $U$, which is the expected behavior for a lower Hubbard band (LHB), and distinguishes it from a low-energy quasi-particle excitation. The existence of a lower Hubbard band is nicely consistent with experimental PES results. Independent measurements on $\mathrm{FeSe}^{6.7}$ revealed a broad feature at a binding energy of about about $-2 \mathrm{eV}$. It was shown in Ref. [6] that the photonenergy dependence of this peak (labeled 'B' in Fig. 1 of Ref. [6]) indicates that it is of Fe- $d$ origin. Given our theoretical results, we propose that the feature observed in Refs. 66.7] in this range of binding energies should be 
interpreted as a lower Hubbard band. To our knowledge, this is indeed the first observation of a lower Hubbard band in iron-based pnictide and chalcogenide superconductors. Indeed, the shoulder at about $-1.5 \mathrm{eV}$ observed in PES for LaFeAsO in Ref. 26 can be explained from band theory. We also note that the position of the Se-p bands agrees well with the experimental peak positions of -6 and $-4 \mathrm{eV}$, corresponding to peaks ' $\mathrm{C}$ ' and ' $\mathrm{D}$ ' in Fig. 1 of Ref. [6].

Our results reveal a marked orbital dependence of the correlation effects, with significantly stronger correlations for the three orbitals $d_{x y}$ and $d_{x z, y z}$, while the $d_{z^{2}}$ and $d_{x^{2}-y^{2}}$ orbitals display weaker correlations. Our conventions for the $(x, y)$ axis are rotated by 45 degrees as compared to the crystallographic axis, so that the $d_{x y}$ orbital in our definition is the one pointing from $\mathrm{Fe}$ to Se. The three orbitals displaying stronger correlations are thus the ones which form the peak of the LDA-DOS (and hence have higher weight at the Fermi level), while the two other ones display a 'pseudo-gap' in the LDA DOS. In Fig. 2, we plot the spectral functions of each Fe- $d$ orbital, calculated for the same parameters as before. It is apparent that the LHB discussed above is visible mostly in the $d_{x y}$ and $d_{x z, y z}$ partial DOS. We have also calculated $Z_{m}=\left.\left(1-\frac{\partial \Sigma}{\partial i \omega}\right)^{-1}\right|_{i \omega \rightarrow 0}$, which - in a Fermi liquid (see however below) - can be interpreted as the quasiparticle weight, and the inverse single-particle lifetime $\operatorname{Im} \Sigma_{m}\left(i 0^{+}\right)$of each orbital. We find that the $d_{z^{2}}$ and $d_{x^{2}-y^{2}}$ orbitals yield larger quasiparticle weights $Z_{z^{2}}=0.38, Z_{x^{2}-y^{2}}=0.47$ and longer lifetimes (corresponding to $-\operatorname{Im} \Sigma\left(i 0^{+}\right)$of order $0.04 \mathrm{eV}$ at $\left.T=290 \mathrm{~K}\right)$. In contrast, a linear fit to the slope of the self-energy for $d_{x y, x z, y z}$ orbitals would yield lower values of the weights $Z_{x y}=0.20$, and $Z_{x z, y z}=0.28$. However, the rather short lifetimes (corresponding to $-\operatorname{Im} \Sigma\left(i 0^{+}\right)_{x y} \simeq 0.19 \mathrm{eV}$ and $\left.-\operatorname{Im} \Sigma\left(i 0^{+}\right)_{x z / y z} \simeq 0.08 \mathrm{eV}\right)$ shed serious doubt on the validity of a coherent quasiparticle interpretation for those orbitals. A further decrease of the temperature from $T=290 \mathrm{~K}$ to $T=190 \mathrm{~K}$ does not reduce $\Sigma\left(i 0^{+}\right)$. In order to identify coherent quasi particles the temperature has to be lower than the width of the quasi-particle, i.e. $T<Z \operatorname{Im} \Sigma\left(i 0^{+}\right)$. Here $Z \operatorname{Im} \Sigma\left(i 0^{+}\right)$for the $d_{x y}$ orbital corresponds to $440 \mathrm{~K}$, which is higher than 290 or $190 \mathrm{~K}$ and, hence, no coherent quasi-particle can be expected. We checked that changing the double counting to "around-mean-field" 24 even increases the incoherence $\left(-\operatorname{Im} \Sigma\left(i 0^{+}\right)_{x z, y z} \approx 0.4\right)$. We elaborate in more detail on this unconventional metallic state further at the end of this paper. The stronger degree of correlations in FeSe, compared to $\mathrm{LaFeAsO}, \frac{18}{18}$, manifests itself also in the partial charges of the Fe- $d$ electrons. Due to electronic correlations, the electron charge diminishes from 6.37 in LDA to 6.07 within LDA+DMFT, which should be compared to the values for $\mathrm{LaFeAsO}$ of 6.40 (LDA) and 6.28 (LDA+DMFT). Please note that these changes do not correspond to any doping effect, since the total electron count of the crystal is still integer. It is merely a redistribution of charges due to changes in the hybridi-
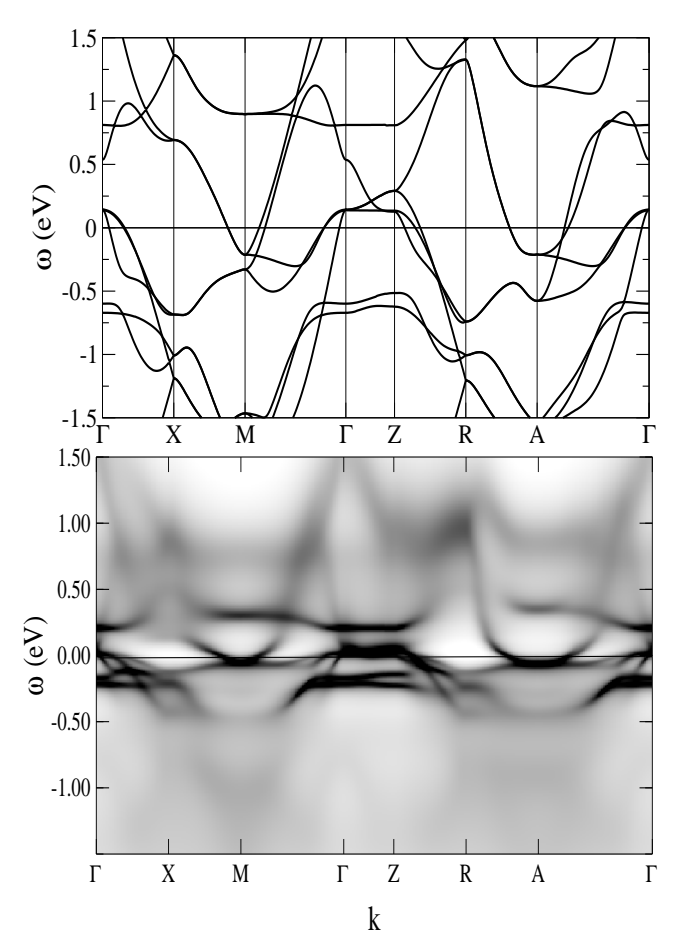

FIG. 3: Comparison of the momentum-resolved spectral function of DFT (top) to the DFT+DMFT results (bottom).

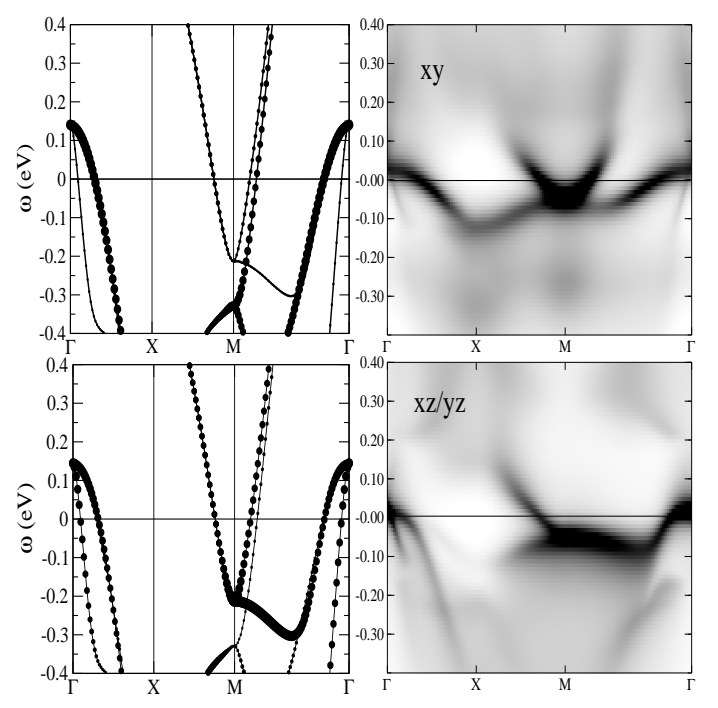

FIG. 4: Comparison of the low-energy spectral function, projected to orbital character, of DFT (left) to the DFT+DMFT results (right). Top row: $d_{x y}$ orbital. Bottom row: Degenerate $d_{x z}, d_{y z}$ orbitals.

sation between the Fe and the ligand atoms.

We have also calculated the momentum-resolved spectral function along high-symmetry directions, displayed in Fig. 3 and compared to the LDA bandstructure. These results can be viewed as predictions for future ARPES experiments on pure FeSe. They can also be 
compared qualitatively with available ARPES data,,$\frac{9,10}{=}$ bearing in mind however that those actually concern the Te-substituted compound $\mathrm{FeSe}_{x} \mathrm{Te}_{1-x}$. The overall renormalization of the bands and the band-width reduction are apparent. The inverse lifetime $(\operatorname{Im} \Sigma)$ increases rapidly with increasing frequency, which results in rather broad structures below $-0.3 \mathrm{eV}$. The DMFT quasiparticle bandstructure cannot be deduced from a mere rescaling of the LDA bandstructure. The strong orbital and energy dependence of the self-energy renormalizes the effective crystal-field splitting between the orbitals, resulting in differential shifts of the quasiparticle bands near the Fermi level. An interesting effect is seen for example near the $\Gamma$ point. In LDA, one can see essentially only two dispersing hole-like bands, the outermost band being quasi-degenerate. By introducing correlations, this degeneracy is lifted, and we can see three hole-like excitations around the $\Gamma$ point. This is qualitatively consistent with ARPES $\stackrel{10}{10}$ We also observe that the hole pockets near the $\Gamma$-point are pushed downwards in energy by correlation effects, while the electron-like ones near the M-point are pushed upwards, in agreement with the ARPES spectra presented in Refs. 9, 10] The orbital character of the LDA+DMFT bands, see Fig. [4 reveals that the outermost band around $\Gamma$ and the electron pocket around $\mathrm{M}$ are dominantly of $d_{x y}$ character, which displays the largest effective mass in our calculations, see above. This is again in qualitative agreement with ARPES, where the heaviest masses were associated to the outermost hole pocket and the electron pocket. Furthermore, the broader feature at around $-0.3 \mathrm{eV}$ is of $d_{z^{2}}$ character, consistent with experiment.

Within the DMFT approach used in the present work, the effective mass enhancement of electrons in a given orbital $m$ is related to the inverse of the weight $Z_{m}$ calculated above. This suggests effective mass enhancements in the range $\sim 2$ for the $x^{2}-y^{2}$ orbital to $\sim 5$ for the $x y$ one. Direct comparison of these values to experiments is difficult in the absence of ARPES data for FeSe. ARPES measurements on $\mathrm{FeSe}_{x} \mathrm{Te}_{1-x}{ }^{9,10}$ have been interpreted as yielding very large effective masses $\left(\mathrm{m}^{*} / \mathrm{m}_{\text {band }}\right.$ between 6 and 23) $\frac{10}{2}$. This is qualitatively consistent with the much larger specific heat coefficient reported for these alloys,$\underline{11}$ in comparison to FeSe $\stackrel{\underline{1}}{\underline{1}}$ It should also be kept in mind that the precise extraction of effective masses (renormalized low-energy Fermi velocities) from ARPES data by comparison to the DFT band structure is subject to rather large uncertainties, especially in view of the orbital-dependent shifts of the bands induced by correlations (see above).

Considering the full rotationally-invariant Hund's coupling 16,17,27 is crucial to describe properly the lowenergy physics of FeSe. Including only density-density interactions drastically suppresses the quasi-particle at low-energies (see Fig. 50), rendering the system much more incoherent for the same value of parameters, as reflected in high scattering rates $-\operatorname{Im} \Sigma\left(i \omega^{+}\right)$in the range $0.5-1.0$. In this case also, lowering the temperature (here, down

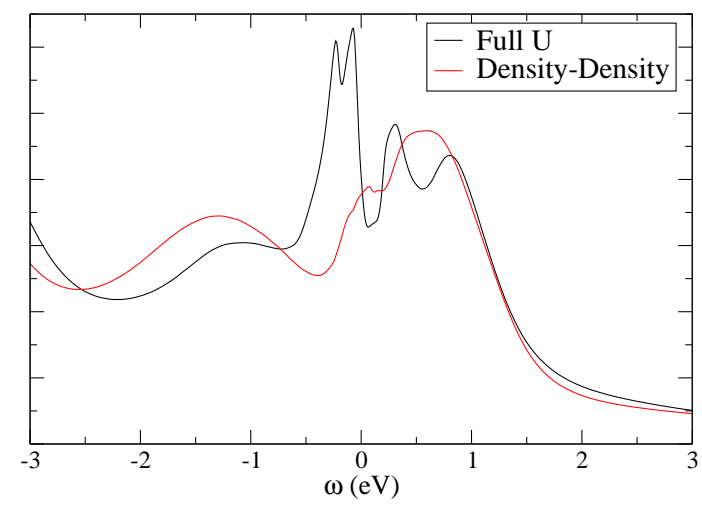

FIG. 5: Difference of the total DOS when calculated using the full 4-index $U$-matrix (black) and density-density interactions (red). For both calculations: $U=4.06 \mathrm{eV}, J=0.91 \mathrm{eV}$.

to $T=50 \mathrm{~K}$ ) does not reduce the scattering rates. An increase of the Coulomb parameters by even $50 \%$ does not drive the system to a Mott-insulator: at $U=6.0 \mathrm{eV}$, $J=1.3 \mathrm{eV}$ there is still finite spectral weight at the Fermi level. We increased $U$ further to $U=10 \mathrm{eV}$ which finally leads to insulating behavior. Taken together, our results for both rotationally invariant and density-density interactions reveal that there is region of the $(U, J)$ parameter space, where the system is in a state which is neither a Fermi-liquid, nor a Mott insulator. This region in parameter space looks similar to the selective localisation found in Refs. [14,28], although we do not find one Mott-localised orbital, but instead three incoherent orbitals. This is also consistent with recent reports within DFT+DMFT calculations based on exact diagonalisation for $\mathrm{LaFeAsO}^{17}$ and $\mathrm{FeSe} .^{29}$

This suggests that FeSe is just beyond the transition from the Fermi-liquid phase to an intermediate badmetallic phase, and could be a realization of the 'spinfreezing' scenario $\stackrel{15.16}{10}$ Indeed, the response to an external magnetic field increases significantly, when this phase is entered (for instance by tuning $J$ ), whichs reveals the formation of localised moments. This appears to be consistent with the rather large values of the resistivity measured even on single-crystal samples $\underline{30}$ and with NMR $^{31}$ and neutron scattering experiments ${ }^{32}$ suggestive of strong spin fluctuations.

\section{CONCLUSIONS}

We have presented strong theoretical and firstprinciples evidence for enhanced electronic correlation effects in FeSe. We have identified a Hubbard satellite in the spectral function, large orbital-dependent mass enhancements ranging from 2 to 5 , and -importantly - large quasi-particle damping effects that may render a Fermi liquid description of the normal state of FeSe invalid over a wide temperature range. Concerning the momentumresolved spectral function, we find very good agreement 
with recent ARPES measurments. In addition to the mass enhancement, we found that a correlation induced crystal-field splitting is important to describe size and position of the hole and electron pockets at the $\Gamma$ and $\mathrm{M}$ point of the Brilloiun zone, respectively. As argued in Ref. [9], a simple scaling of the LDA bands is not enough to account for the measured electronic structure. The present orbital-sensitive non-Fermi liquid behavior further indicated by their sensitivity to the Hund's coupling may serve for understanding the interplay between the spin-charge-orbital dynamics and superconductivity in iron superconductors.

\section{Acknowledgments}

MA, SB, and AG acknowledge discussions with V. Vildosola, L. Pourovskii, M. Ferrero, O. Parcollet, A. Lieb- sch, H. Ding, L. Craco, L. de' Medici and D. van der Marel. TM and MI thank discussions with R. Arita, K. Nakamura and T. Misawa. This research was supported in part by the Agence Nationale de la Recherche under grant CORRELMAT, of IDRIS/GENCI (project 101393), the National Science Foundation under Grant No. PHY05-51164, and (MA) the Austrian Science Fund (FWF) under grant J2760. The hospitality of the Kavli Institute for Theoretical Physics is gratefully acknowledged.
1 F.-C. Hsu, J.-Y. Luo, K.-W. Yeh, T.-K. Chen, T.-W. Huang, P. M. Wu, Y.-C. Lee, Y.-L. Huang, Y.-Y. Chu, D.-C. Yan, et al., Proc. Nat. Acad. Sci. U.S.A. 105, 14262 (2008).

2 Y. Mizuguchi, F. Tomioka, S. Tsuda, T. Yamaguchi, and Y. Takano, Appl. Phys. Lett. 93, 152505 (2008).

${ }^{3}$ G. Garbarino, A. Sow, P. Lejay, A. Sulpice, P. Toulemonde, M. Mezouar, and M. Núñez-Regueiro, Europhys. Lett. 86, 27001 (2009).

4 S. Margadonna, Y. Takabayashi, Y. Ohishi, Y. Mizuguchi, Y. Takano, T. Kagayama, T. Nakagawa, M. Takata, and K. Prassides, Phys. Rev. B 80, 064506 (2009).

${ }^{5}$ K.-W. Yeh, T.-W. Huang, Y.-L. Huang, T.-K. Chen, F.-C. Hsu, P. M. Wu, Y.-C. Lee, Y.-Y. Chu, C.-L. Chen, J.-Y. Luo, et al., Europhys. Lett. 84, 37002 (2008).

${ }^{6}$ R. Yoshida, T. Wakita, H. Okazaki, Y. Mizuguchi, S. Tsuda, Y. Takano, H. Takeya, K. Hirata, T. Muro, M. Okawa, et al., J. Phys. Soc. Jpn. 78, 034708 (2009).

7 A. Yamasaki, S. Imada, K. Takase, T. Muro, Y. Kato, H. Kobori, A. Sugimura, N. Umeyama, H. Sato, Y. Hara, et al., arXiv/0902.3314.

8 Y. Xia, D. Qian, L. Wray, D. Hsieh, G. F. Chen, J. L. Luo, N. L. Wang, and M. Z. Hasan, Phys. Rev. Lett. 103, 037002 (2009).

9 K. Nakayama, T. Sato, P. Richard, T. Kawahara, Y. Sekiba, T. Qian, G. F. Chen, J. L. Luo, N. L. Wang, H. Ding, et al., arXiv/0907.0763.

10 A. Tamai, A. Y. Ganin, E. Rozbicki, J. Bacsa, W. Meevasana, P. D. C. King, M. Caffio, R. Schaub, S. Margadonna, K. Prassides, et al., Phys. Rev. Lett. 104 (2010).

11 B. C. Sales, A. S. Sefat, M. A. McGuire, R. Y. Jin, D. Mandrus, and Y. Mozharivskyj, Phys. Rev. B 79, 094521 (2009).

12 A. Subedi, L. Zhang, D. J. Singh, and M. H. Du, Phys. Rev. B 78, 134514 (2008).

13 T. Miyake, K. Nakamura, R. Arita, and M. Imada, J. Phys. Soc. Jpn. 79044705 (2010).

14 L. Craco, M. S. Laad, and S. Leoni, arXiv/0910.3828.

15 P. Werner, E. Gull, M. Troyer, and A. J. Millis, Phys. Rev.
Lett. 101, 166405 (2008).

16 K. Haule and G. Kotliar, New J. Phys. 11, 025021 (2009).

17 H. Ishida and A. Liebsch, Phys. Rev. B 81 (2010).

18 M. Aichhorn, L. Pourovskii, V. Vildosola, M. Ferrero, O. Parcollet, T. Miyake, A. Georges, and S. Biermann, Phys. Rev. B 80, 085101 (2009).

19 P. Blaha, K. Schwarz, G. Madsen, D. Kvasnicka, and J. Luitz, WIEN2k, An augmented Plane Wave + Local Orbitals Program for Calculating Crystal Properties (Techn. Universitat Wien, Austria, ISBN 3-9501031-1-2., 2001).

20 S. Margadonna, Y. Takabayashi, M. T. McDonald, K. Kasperkiewicz, Y. Mizuguchi, Y. Takano, A. N. Fitch, E. Suard, and K. Prassides, Chem. Commun. 43, 5607 (2008).

21 P. Werner, A. Comanac, L. de' Medici, M. Troyer, and A. J. Millis, Phys. Rev. Lett. 97, 076405 (2006).

${ }^{22}$ F. Aryasetiawan, M. Imada, A. Georges, G. Kotliar, S. Biermann, and A. I. Lichtenstein, Phys. Rev. B 70, 195104 (2004).

23 T. Miyake, L. Pourovskii, V. Vildosola, S. Biermann, and A. Georges, J. Phys. Soc. Jpn. 77, 99 (2008).

24 E. R. Ylvisaker, W. E. Pickett, and K. Koepernik, Phys. Rev. B 79, 035103 (2009).

${ }^{25}$ K. S. D. Beach, arXiv/0403055.

26 W. Malaeb, T. Yoshida, T. Kataoka, A. Fujimori, M. Kubota, K. Ono, H. Usui, K. Kuroki, R. Arita, H. Aoki, et al., J. Phys. Soc. Jpn. 77, 093714 (2008).

27 T. Pruschke and R. Bulla, Eur. Phys. J. B 44, 217 (2005).

28 L. de Medici, S. R. Hassan, and M. Capone, J. Supercond. Nov. Magn. 22, 535 (2009).

29 A. Liebsch and H. Ishida, arxiv/1004.2851.

30 D. Braithwaite, B. Salce, G. Lapertot, F. Bourdarot, C. Marin, D. Aoki, and M. Hanfland, J. Phys. Cond. Mat. 21, 232202 (2009).

31 T. Imai, K. Ahilan, F. L. Ning, T. M. McQueen, and R. J. Cava, Phys. Rev. Lett. 102, 177005 (2009).

32 M. D. Lumsden, A. D. Christianson, E. A. Goremychkin, S. E. Nagler, H. A. Mook, M. B. Stone, D. L. Abernathy, T. Guidi, G. J. MacDougall, C. de la Cruz, et al., Nature Physics 6, 182 (2010). 